\title{
Alkaline Phosphatase: Placental and Tissue-nonspecific Isoenzymes Hydrolyze Phosphoethanolamine, Inorganic Pyrophosphate, and Pyridoxal 5'-Phosphate Substrate Accumulation in Carriers of Hypophosphatasia Corrects during Pregnancy
}

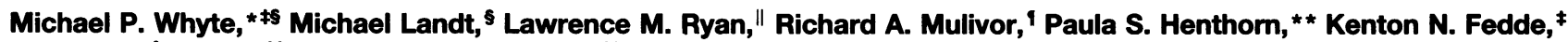 \\ J. Dennis Mahuren, \# and Stephen P. Coburn \# \\ ${ }^{*}$ Metabolic Research Unit, Shriners Hospital for Crippled Children, St. Louis, Missouri 63131; ${ }^{\ddagger}$ Division of Bone and Mineral Diseases \\ at The Washington University Medical Center, The Jewish Hospital of St. Louis, St. Louis, Missouri 63110; ${ }^{\S}$ Department of Pediatrics, \\ Washington University School of Medicine, St. Louis, Missouri 63110; "Department of Rheumatology, The Medical College of Wisconsin, \\ Milwaukee, Wisconsin 53226; 'Coriell Institute For Medical Research, Camden, New Jersey 08103; ** Section of Medical Genetics, \\ University of Pennsylvania School of Veterinary Medicine, Philadelphia, Pennsylvania 19104; and ${ }^{\ddagger \ddagger}$ Fort Wayne State Developmental \\ Center, Fort Wayne, Indiana 46835
}

\section{Abstract}

Hypophosphatasia features selective deficiency of activity of the tissue-nonspecific (liver/bone/kidney) alkaline phosphatase (ALP) isoenzyme (TNSALP); placental and intestinal ALP isoenzyme (PALP and IALP, respectively) activity is not reduced. Three phosphocompounds (phosphoethanolamine [PEA], inorganic pyrophosphate [PPi], and pyridoxal 5 '-phosphate [PLP]) accumulate endogenously and appear, therefore, to be natural substrates for TNSALP. Carriers for hypophosphatasia may have decreased serum ALP activity and elevated substrate levels.

To test whether human PALP and TNSALP are physiologically active toward the same substrates, we studied PEA, PPi, and PLP levels during and after pregnancy in three women who are carriers for hypophosphatasia. Hypophosphatasemia corrected during the third trimester because of PALP in maternal blood. Blood or urine concentrations of PEA, PPi, and PLP diminished substantially during that time. After childbirth, maternal circulating levels of PALP decreased, and PEA, PPi, and PLP levels abruptly increased. In serum, unremarkable concentrations of IALP and low levels of TNSALP did not change during the study period.

We conclude that PALP, like TNSALP, is physiologically active toward PEA, PPi, and PLP in humans. We speculate from molecular/crystallographic information, indicating significant similarity of structure of the substrate-binding site of ALPs throughout nature, that all ALP isoenzymes recognize these same three phosphocompound substrates. (J. Clin. Invest. 1995. 95:1440-1445.) Key words: enzyme • mineralization • rickets $\bullet$ phosphocompounds $\bullet$ vitamin $\mathbf{B}_{6}$

This work was presented in part at the 14th Annual Scientific Meeting of the American Society for Bone and Mineral Research in Minneapolis, MN on 30 September to 4 October 1992 (1992. J. Bone Miner. Res. 7[Suppl. 1]:S-155).

Address correspondence to Michael P. Whyte, MD, Medical Director, Metabolic Research Unit, Shriners Hospital for Crippled Children, 2001 South Lindbergh Boulevard, St. Louis, MO 63131. Phone: 314432-3600; FAX: 314-432-2930.

Received for publication 1 August 1994 and in revised form 25 October 1994.

\footnotetext{
J. Clin. Invest.

(c) The American Society for Clinical Investigation, Inc.

0021-9738/95/04/1440/06 \$2.00

Volume 95, April 1995, 1440-1445
}

\section{Introduction}

Alkaline phosphatases (ALPs) ${ }^{1}$ are ubiquitous in nature (1). Nevertheless, the physiological role(s) of these enzymes is poorly understood (2). Their biochemical designation (orthophosphoric monoester phosphohydrolase [alkaline optimum]: EC 3.1.3.1) reflects catalytic properties characterized in vitro (1).

In humans, at least four genes encode ALP isoenzymes (3). Three ALP genes at chromosome 2q34-37 are expressed in essentially a tissue-specific manner and produce a placental, placental-like, and intestinal ALP isoenzyme (PALP, PLALP, and IALP, respectively). The fourth ALP gene, at chromosome $1 \mathrm{p} 36.1-34$, encodes a family of proteins that differ from one another only by posttranslational modification; these secondary ALP isoenzymes are present throughout the body, but individually are most abundant in hepatic, skeletal, and renal tissue (4). Accordingly, they are collectively called liver/bone/kidney or tissue-nonspecific ALP (TNSALP) (1-4). At the amino acid level, the tissue-specific ALP isoenzymes are 86-98\% identical to one another $(5,6)$, but $52-56 \%$ identical when compared with TNSALP (7).

Insight concerning the physiological function of TNSALP in humans has come from investigation of hypophosphatasia $(2,8)$, a rare heritable metabolic bone disease characterized biochemically by a deficiency of TNSALP activity (9). Clinical features include rickets in children or osteomalacia in adults and premature loss of teeth because of defective formation of dental cementum (9). In hypophosphatasia, three phosphocompounds (phosphoethanolamine [PEA], inorganic pyrophosphate [PPi], and pyridoxal 5'-phosphate [PLP]) accumulate endogenously and appear, therefore, to be natural substrates for TNSALP $(8,9)$. PPi is an inhibitor of calcification and, accordingly, increased levels could account for the skeletal disease $(8,10)$. Identification of 12 missense mutations to date within the TNSALP gene (11-13 and Henthorn, P. S., M. Raducha, V. Fimiani, K. N. Fedde, and M. P. Whyte, manuscript in preparation) associated with hypophosphatasia and a transfection study, which showed that one of these nucleotide changes destroyed the enzyme's catalytic action (11), establish that this heritable metabolic bone disease is an inborn error of

1. Abbreviations used in this paper: ALP, alkaline phosphatase; IALP, intestinal ALP isoenzyme; PALP, placental ALP isoenzyme; PEA, phosphoethanolamine; PLALP, placental-like ALP isoenzyme; PLP, pyridoxal 5'-phosphate; PPi, inorganic pyrophosphate; PS, phosphoserine; TNSALP, tissue-nonspecific ALP isoenzyme. 
metabolism and that TNSALP has a critical role in skeletal mineralization and formation of dentition in humans (8).

Although investigation of hypophosphatasia has provided considerable insight concerning the biological role of TNSALP (8) in biomineralization, inborn errors of PALP, PLALP, and IALP biosynthesis have not been described, and the function of these tissue-specific ALP isoenzymes is less well understood (1). ALP gene knockout models in animals have not yet been reported. Furthermore, the tissue specificity and organization of the human ALP genes is not homologous in mice (1), and murine knockout models will not be completely analogous for exploring human ALP isoenzyme function.

X-ray crystallographic study of Escherichia coli ALP (14) together with comparisons of the ALP proteins of a variety of organisms $(15,16)$ indicate that the core structure, active site, and catalytic mechanism of this enzyme have been significantly conserved throughout evolution. Accordingly, we hypothesized that PALP and TNSALP (and by extrapolation all four of the ALP isoenzymes in humans) possess similar substrate specificity. We tested this hypothesis by studying throughout and after pregnancy women who were hypophosphatasia carriers (i.e., TNSALP-deficient). In the course of the investigation, we quantitated serum ALP isoenzymes, plasma PLP, and urinary PEA and PPi. We predicted that levels of these three phosphocompounds would diminish during the latter stages of gestation when significant amounts of PALP are synthesized endogenously if, in fact, the substrate specificity of PALP is similar to TNSALP.

\section{Methods}

Subjects. Subjects A, B, and C each had had a child with either the moderately severe childhood form or more mild odonto form of hypophosphatasia (9). Each woman had participated in a screening investigation of their kindred (before the pregnancy study described herein) and manifested biochemical findings consistent with carrier status for hypophosphatasia (hypophosphatasemia and elevated plasma PLP levels), but did not have clinical evidence of hypophosphatasia. Respectively, they had serum total ALP activity (assayed in different clinical laboratories) of $8 \mathrm{IU} /$ liter (35-100 normal), $19 \mathrm{IU} /$ liter (28-91 nor$\mathrm{mal}$ ), and $11 \mathrm{IU} /$ liter (35-100 normal); plasma PLP concentrations were 624,136 , and $164 \mathrm{nM}$ (30-110 normal). These same biochemical abnormalities were also present in a few of their first-degree relatives, several of whom had medical histories suggestive of mild skeletal/ dental manifestations of hypophosphatasia. However, the husbands of the study volunteers and the husbands' relatives had serum total ALP activities and plasma PLP levels that were unremarkable and they did not have clinical features of hypophosphatasia. Subsequent molecular studies of leukocyte DNA, using allele-specific oligonucleotide hybridization of the 12 known TNSALP missense mutations associated with hypophosphatasia (11-13 and Henthorn, P. S., M. Raducha, V. Fimiani, K. N. Fedde, and M. P. Whyte, manuscript in preparation), identified subject $A$ and her affected children to carry a 1309-T (cDNA) base change in one TNSALP allele. The affected child of subject $B$ is negative for 11 of the TNSALP mutations searched for to date. Molecular investigation of subject $C$ is recently underway. Thus, our cumulative information provides no evidence that the husbands are carriers for hypophosphatasia. Although further study is necessary, the disorder may have been transmitted from the mothers, consistent with several reports of autosomal dominant transmission with variable penetrance for mild forms of this inborn error of metabolism (17-20).

Specimen collection. After the review of the provocative but limited peripartum data from our pilot study of subject $C$, we conducted complete prospective investigations of subjects $\mathrm{A}$ and $\mathrm{B}$ beginning in early pregnancy and ending several months after childbirth.

After informed consent from each volunteer, at that time of monthly prenatal and postnatal checkups, their obstetrician froze a specimen of serum and heparinized plasma as well as aliquots from 24-h urine collections. Throughout the hospitalizations for childbirth, spot or 24$\mathrm{h}$ urine collections and blood samples were obtained. Specimens were shipped on dry ice periodically during the study, stored at $-40^{\circ} \mathrm{C}$, and soon after assayed in duplicate. When the pregnancy was discovered, per routine practice by obstetricians, each woman received prenatal multivitamins containing $4 \mathrm{mg}$ of vitamin $B_{6}$ as pyridoxine that were continued throughout the study. Umbilical cord blood was obtained from each newborn to screen for biochemical changes of hypophosphatasia.

Biochemical assays. Serum total ALP activity was assayed kinetically in the laboratory of the Metabolic Research Unit, Shriners Hospital for Crippled Children (St. Louis, MO) with a spectrophotometer (DU6; Beckman Instruments, Inc., Brea, CA) according to the method of McComb, Bowers, and Posen (1) at pH 10.1 using $p$-nitrophenylphosphate substrate; normal range ( \pm 2 SD mean) for adults was 28-91 IU/ liter and for children 80-342 IU/liter. Creatinine in urine was quantitated with this same instrument. Urinary PEA was measured at the Metabolic Genetics Laboratory of the Department of Pediatrics, Washington University School of Medicine (St. Louis, MO) using a Beckman 7300 analyzer and reagents and procedures provided by the manufacturer. This method also quantitated urinary phosphoserine (PS) (see below). Urinary PPi was quantitated at The Medical College of Wisconsin (Milwaukee, WI) by a modification of the radiometric uridine diphosphoglucose pyrophosphorylase method of Cheung and Suhadolnik (21). Plasma PLP was assayed at the Fort Wayne State Developmental Center (Fort Wayne, IN) by the cation-exchange, high-pressure liquid chromatography procedure developed in that laboratory (22); normal range was 30-110 nM. PALP and the bone form of TNSALP in serum were estimated at the Metabolic Research Unit, Shriners Hospital for Crippled Children (St. Louis, MO) by heat inactivation at $65^{\circ} \mathrm{C}$ for 5 min or $56^{\circ} \mathrm{C}$ for $15 \mathrm{~min}$, respectively (1). PALP, TNSALP, and IALP were quantitated at the Coriell Institute for Medical Research (Camden, NJ) by stereospecific amino acid inhibition of ALP isoenzymes, standard heat denaturation studies, and immunoprecipitation using monoclonal antibodies specific for human ALP isoenzymes (23).

\section{Results}

The biochemical findings during each of the three pregnancies were similar and are illustrated in Fig. 1.

$A L P$ isoenzymes during pregnancy. As described previously, each woman had been shown to be hypophosphatasemic before her pregnancy. Subjects A and B showed gradual increases in serum total ALP activity (Fig. 1, row $I$ of panels) beginning at about 5 mo of gestation. Relatively rapid increments occurred during the third trimester until the time of fullterm delivery (designated by arrows). Compared with the normal range for serum total ALP activity in normal adults in St. Louis, these increases corrected subnormal (nonpregnant) levels by the sixth month of gestation. At $\sim 8$ mo of gestation, the serum total ALP activity levels were elevated. Subject C, who was studied only at delivery and after childbirth, had values for serum total ALP activity that had risen into the normal adult range at the time of parturition.

The increases in serum total ALP in subjects A and B were due exclusively to PALP (Fig. 2). Subject A, at 4 mo of pregnancy, had PALP comprise $22 \%$ of her serum total ALP. However, during the month before delivery, $>90 \%$ of her serum total ALP was PALP. In fact, at the time of parturition, total ALP activity was $92 \%$ PALP, $7 \%$ TNSALP, and $1 \%$ IALP. Quantitation of TNSALP showed subnormal and unchanged values during and after the pregnancy; IALP was always a very small percentage of the total. Subject B manifested $16 \%$ of her serum total ALP as PALP at 4.5 mo of pregnancy. 1 mo before delivery her serum total ALP activity was $113 \mathrm{IU} /$ liter (normal 


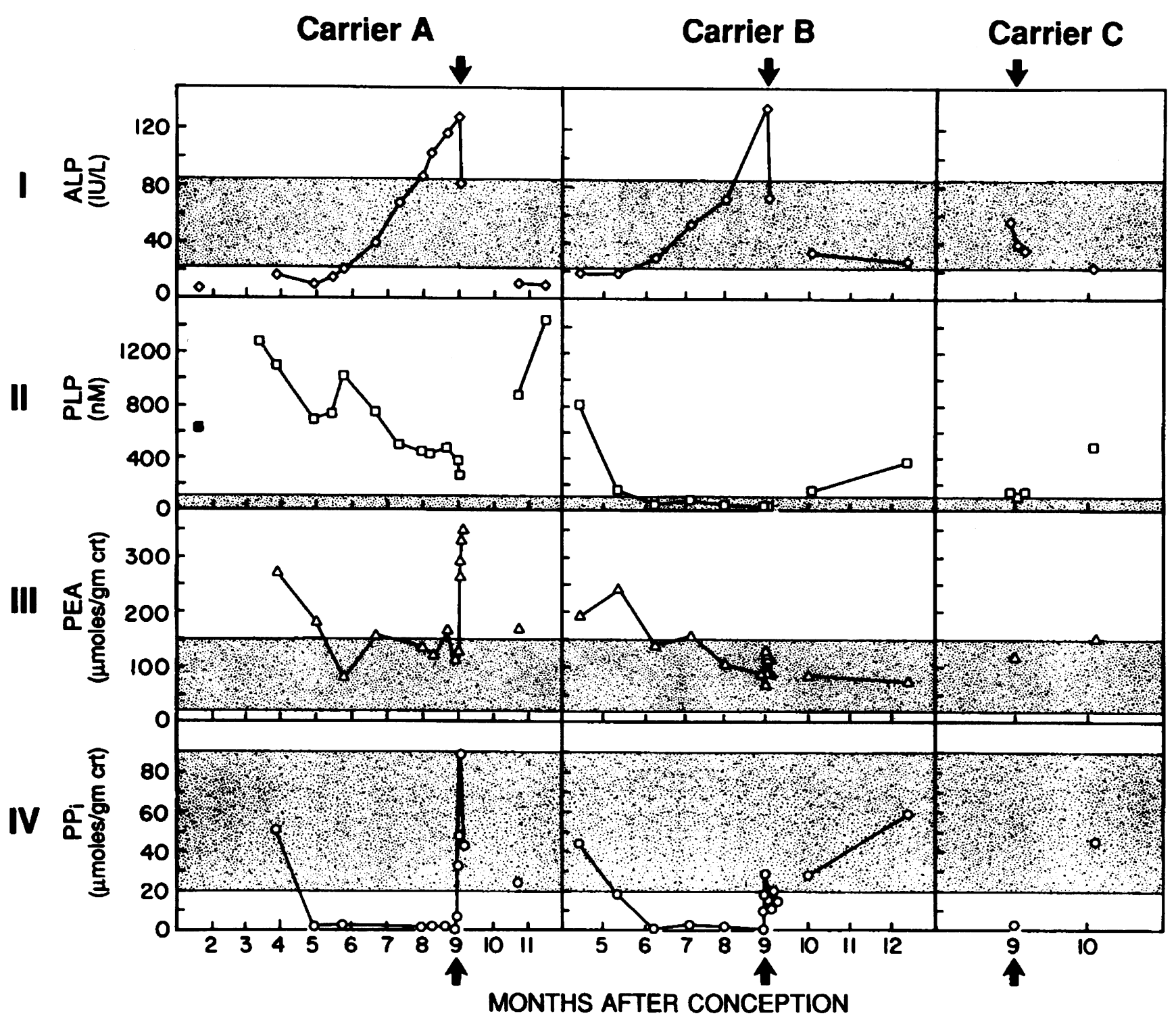

Figure 1. Biochemical changes in three carriers for hypophosphatasia during and after pregnancy. Columns $A, B$, and $C$ depict findings for subjects A, B, and C; rows I-IV show serum total ALP activity, plasma PLP concentration, and urinary PEA and PPi levels, respectively. The abscissa depicts months since conception. Arrows indicate the time of childbirth. Stippled areas show ranges $( \pm 2$ SD mean) for normal men and women (see Methods). $\square$ indicates a plasma PLP level before prenatal vitamins were taken. Responses are shown with discontinuous lines when the number of samples precluded depiction of precise temporal sequences. Note the substantial increases in ALP activity and decreases in PLP, PEA, and PPi levels during the later stages of pregnancy and abrupt increases in phosphocompounds at the time of delivery. crt, creatinine.

adult range 68-206 IU/liter) (74\% PALP, 24\% TNSALP, and $2 \%$ IALP). The value then rose to 202 IU/liter (81\% PALP, $19 \%$ TNSALP, and $0 \%$ IALP) at delivery. Subject $\mathrm{C}$ had a predominance $(73 \%)$ of heat-stable $\left(65^{\circ} \mathrm{C} \times 5 \mathrm{~min}\right)$ ALP (i.e., PALP) the day before delivery.

$P E A, P P i$, and PLP during pregnancy. Subject A had an elevated level of plasma PLP of $600 \mathrm{nM}$ (30-110 normal) shortly after conception. After initiation of prenatal vitamin treatment early in her pregnancy (and then continued daily throughout the study), the level was significantly increased, as expected, ${ }^{2}$ to $\sim 1,300 \mathrm{nM}$ at 2.5 to 3 mo of gestation. Subse-

2. Patients with hypophosphatasia (2), as well as Canadian Mennonite carriers for hypophosphatasia (24), have an exaggerated plasma PLP level response when given an oral pyridoxine challenge. quently, there were sizable decreases in plasma or urine levels of PLP, PEA, and PPi (Fig. 1) even before PALP had normalized serum total ALP activity in her circulation. During the third trimester of pregnancy, plasma PLP concentrations (Fig. 1 , row $I I$ ) had nearly corrected despite vitamin supplementation, urinary PEA levels (Fig. 1, row III) were normal, and urinary PPi levels (Fig. 1, row $I V$ ) became undetectable. Very similar changes occurred for subject B who normalized her plasma PLP concentrations and urine PEA levels and had urinary PPi levels below the limits of detection during the third trimester of pregnancy.

Postpartum findings. After parturition, serum total ALP activity $(\sim$ PALP) decreased with a measured half-life for subjects $\mathrm{A}, \mathrm{B}$, and $\mathrm{C}$ of 1.8, 2.1, and $4.5 \mathrm{~d}$, respectively (Fig. 1). Urinary PEA and PPi levels, however, were found to rise abruptly. Elevated urinary PEA levels and detectable PPi con- 


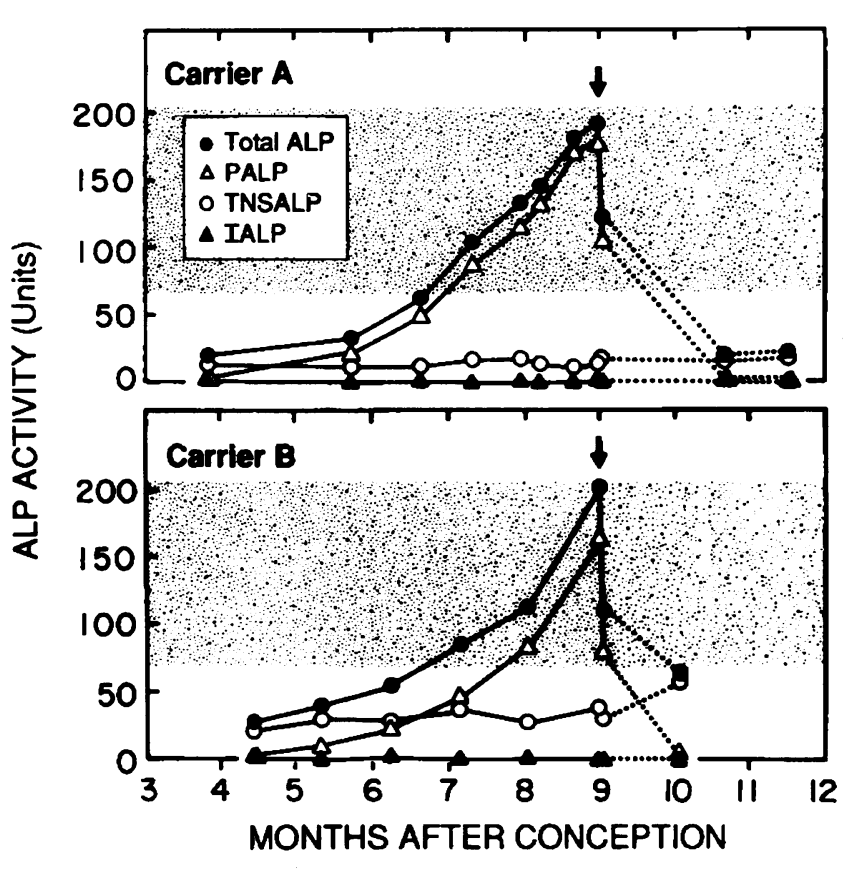

Figure 2. Serum ALP isoenzyme levels in two carriers for hypophosphatasia during and after pregnancy. The key in the top panel defines the individual ALP isoenzymes studied. The abscissa depicts months since conception. Arrows indicate the time of childbirth. Stippled areas show ranges ( \pm 2 SD mean) for serum total ALP activity (IU/liter) for normal men and women (see Methods). Responses are shown with discontinuous lines when the number of samples precluded depiction of precise temporal sequences. Note that the increase in serum total ALP during pregnancy was accounted for by PALP; TNSALP and IALP remained essentially unchanged throughout the study.

centrations were noted in subject $\mathrm{A}$ at a time when her serum total ALP was still in the normal range. Especially high plasma PLP concentrations were demonstrated for subjects $\mathrm{A}$ and $\mathrm{C}$ several months after childbirth when they were once again clearly hypophosphatasemic and prenatal vitamins were continued.

Offspring. Subject A delivered a boy whose cord serum revealed hypophosphatasemia (total ALP activity $15 \mathrm{IU} /$ liter) and an elevated plasma PLP concentration of 1,989 nM (previously, we had assayed cord blood from two newborn controls [mothers presumably receiving vitamins] whose serum total ALP activities were 109 and 135 IU/liter, and plasma PLP concentrations were 180 and $275 \mathrm{nM}$ [25]). Now, $7 \mathrm{yr}$ old, like his affected older brother, he has had premature loss of deciduous teeth and has subtle rachitic changes on skeletal radiographs. Therefore, he mildly manifests the childhood form of hypophosphatasia. Molecular analysis revealed that he (like his mother, brother, sister, and several other maternal relatives with biochemical findings consistent with hypophosphatasia) carries the 1309-T TNSALP missense mutation. Subject A, now $46 \mathrm{yr}$ old, appears recently to be developing clinical evidence of hypophosphatasia with recurrent poorly healing metatarsal stress fractures and failure of bone implants to preserve her dentition.

Subject B delivered a boy. His older sister has childhood hypophosphatasia. Total ALP activity in his cord serum was $133 \mathrm{IU} /$ liter. There was $10 \%$ residual activity after heat inactivation at $56^{\circ} \mathrm{C} \times 15 \mathrm{~min}$, suggesting a predominance of the bone form of TNSALP. More detailed analysis of his cord serum showed a total ALP activity of 249 IU/liter (normal range in children 3-15 yr old, 178-556 IU/liter) with an unremarkable profile of $83 \%$ TNSALP, $1 \%$ PALP, and $16 \%$ IALP. His plasma PLP concentration of $147 \mathrm{nM}$ was normal (25). He is now $7 \mathrm{yr}$ old, well, and has not had premature loss of teeth.

Subject C, whose first daughter had odontohypophosphatasia, delivered a girl who had low cord serum ALP activity at the Metabolic Research Unit of 33 IU/liter and a high plasma PLP level of 1,237 nM. However, at $7.5 \mathrm{yr}$ of age, this child is in excellent health and age-appropriately is just beginning to shed her deciduous teeth. She takes no multivitamins. Recent reassay of the serum total ALP activity and plasma PLP level of this child and her mother showed values consistent with carrier status for both individuals, i.e., $49 \mathrm{IU} /$ liter and $265 \mathrm{nM}$; $15 \mathrm{IU} /$ liter and $55 \mathrm{nM}$ (now normal), respectively.

\section{Discussion}

Although the precise pathogenesis of hypophosphatasia is uncertain, characterization of the clinical, biochemical, pathological, and molecular features of this inborn error of metabolism confirms, in humans, Robert Robison's hypothesis of 1923 that ALP functions importantly in skeletal mineralization (26). Failure of TNSALP to hydrolyze PPi, an inhibitor of biomineralization, offers a plausible explanation for the associated skeletal disease $(8,10)$. Hypophosphatasia, however, raises intriguing questions about the physiological role of TNSALP elsewhere in the body, because organs other than the skeleton that are also TNSALP-rich (e.g., liver, kidney, adrenal glands) do not significantly malfunction $(2,8)$. Indeed, after considerable investigative effort, a great variety of physiological roles has been suggested not only for TNSALP, but for all four ALP isoenzymes in humans (1) (for review see reference 8 ). The absence of human models for PALP, PLALP, or IALP deficiency has hindered our understanding of the role of the three tissue-specific ALP isoenzymes $(1,4,27)$. Furthermore, separate genes for PALP and PLALP are found only in higher primates (1); thus, development of animal models is problematic.

The decreases in blood and urine levels of PEA, PPi, and PLP that we observed during the pregnancies of three women who are carriers for hypophosphatasia are consistent with our aforementioned speculation, based upon comparable substrate binding site structures throughout nature $(15,16)$, that all ALP isoenzymes in humans have at least overlapping specificities for physiological substrates. Our findings show that PALP acts in vivo to hydrolyze the same substrates as TNSALP, i.e., PEA, $\mathrm{PPi}$, and PLP which are sensitive biochemical markers for hypophosphatasia (28). In our study subjects, elevated plasma PLP concentrations and increased urinary PEA levels normalized and urinary PPi became undetectable during the third trimester of pregnancy when PALP was present endogenously as shown by significant PALP levels in serum. Reviewed below, these biochemical observations seem attributable to PALP (rather than some other phosphatase), and specifically to PALP in the placenta itself, not PALP in the circulation.

In human tissues, all forms of ALP are primarily bound to the external surface of cells $(29,30)$. ALP isoenzyme patterns in the circulation are believed to reflect their source in specific organs (1). For healthy adults, approximately equal amounts of the bone and liver forms of the TNSALP isoenzyme comprise most of the ALP in serum $(23,31)$; IALP makes up only a small percentage of the total $(1,23)$. PLALP and PALP may 
appear with certain malignancies $(6,27)$, but are normally difficult to detect except for PALP during pregnancy (23).

PALP is expressed from the genome of the fetus. Early during the first trimester of pregnancy, PALP in the chorionic villus is essentially absent (32). PALP then appears predominantly on the plasma membrane of the maternal surface of syncytiotrophoblast microvilli $(33,34)$. Changes in maternal serum ALP isoenzyme levels during gestation have been thoroughly studied (35-37). For uneventful pregnancies, serum total ALP activity rises gradually between the first and second trimesters, then rapidly increases further during the third trimester. The increments are due both to PALP and to some increase in the bone form of TNSALP (38). In their comprehensive review Alkaline Phosphatase, McComb, Bowers, and Posen (1) cite the experimental evidence that "established beyond doubt that the enhanced level of serum ALP in pregnant women is due predominantly to the enrichment of the circulation with enzyme of placental origin"' (35-38). Although approximately a doubling of the bone form of TNSALP in serum occurs between the second and third trimesters of pregnancy, which persists for $6 \mathrm{wk}$ after delivery $(38,39)$, the changes in PALP are greater. During the third trimester, normally $\sim 40-67 \%$ of the total ALP in maternal serum is PALP (36). ${ }^{3}$

Since expression of PALP is controlled by the fetal genome, one would not expect a defect in PALP biosynthesis in hypophosphatasia (33). Women who are affected by, or are carriers for, hypophosphatasia would, therefore, be excellent models for exploring the physiological effect of PALP. PALP appearance endogenously would occur on a background of TNSALP deficiency and with little (if any) change in other ALPs. In fact, we observed unremarkable temporal and quantitative aspects of PALP biosynthesis during the closely followed pregnancies of our study subjects. An especially high percentage of their serum total ALP at delivery was PALP, i.e., 92, 81, and 73\% in subjects $A, B$, and $C$, respectively. We found no change in their deficient TNSALP or unremarkable IALP levels in serum.

Our ability to assess the impact of endogenous biosynthesis of PALP per se seemed especially good in subject A. Her newborn was affected by hypophosphatasia as shown by his cord blood that had low serum ALP activity and elevated plasma PLP concentrations and his subsequent clinical course. Changes observed in her endogenous PEA, PPi, and PLP levels were, therefore, unlikely to have been influenced by fetal TNSALP. In fact, as exemplified by PLP (41), phosphorylated compounds do not readily cross the fetal/maternal placental barrier, and PALP does not have access to the fetal circulation (42).

The placenta has complex endocrine functions and synthesizes placental-specific enzymes $(43,44)$. Conceivably, biosynthesis of some phosphatase other than PALP by the placenta could have accounted for the observed changes in PEA, PPi, and PLP. However, the temporal relationship between the changes in serum PALP levels and the decreases in PEA, PPi, and PLP levels was consistent with hydrolysis of these phosphocompounds by PALP.

Evidence that PALP within the placenta itself, rather than in the circulation, caused the observed changes in PEA, PPi, and

3. During pregnancy there is typically some increase in neutrophil ALP activity, which has been shown to be a form of TNSALP (40), but this ALP constitutes only a small amount of the total ALP activity in the circulation, and can be subnormal in all clinical forms of hypophosphatasia (9).
PLP comes from examination of the timing of the biochemical changes. The half-life for PALP infused into volunteers has been reported to be $\sim 7 \mathrm{~d}(45)$. In normal pregnant women, a monoclonal antibody-based, solid-phase, enzyme-binding assay for serum PALP showed an immediate postpartum drop within $4 \mathrm{~d}$ to values approaching nonpregnant levels (37). Postpartum, the serum PALP of our study subjects decreased with calculated half-lives of 1.8-4.5 d. We observed, however, that urinary PEA and PPi levels, and perhaps plasma PLP concentrations as well, increased more abruptly than circulating PALP dissipated at the time of delivery. Although the rapidity of the changes was somewhat difficult to illustrate in Fig. 1, assay of spot urine collections demonstrated a very rapid increase in urinary PEA and PPi levels within the first day postpartum. The relatively gradual decrease of PALP levels in the circulation over several days did not reflect these changes. Indeed, at a time when serum total ALP was still in the normal range, urinary levels of PEA and PPi had clearly increased. Sudden physiological expulsion of the placenta and its PALP during parturition seemed to explain the rapid reaccumulation of these ALP substrates. Similarly, early in the pregnancies, when relatively small amounts of PALP were detectable in serum, there already had been a significant decrement in plasma PLP concentrations into the normal range, partial correction of urinary PEA levels, and almost virtual disappearance of PPi from the urine. These cumulative observations are consistent with reports that ALP in situ, rather than in the circulation, is physiologically active $(2,8,9$, 45-49).

Of interest, our previous studies of a large kindred with the adult form of hypophosphatasia suggested that phosphoserine (PS) could be a physiological substrate for TNSALP. In hypophosphatasemic subjects, there was a negative correlation between urinary PS levels and serum levels of the liver form of TNSALP $(17,31)$. However, urinary concentrations of PS in subjects $\mathrm{A}, \mathrm{B}$, and $\mathrm{C}$ were not increased and remained essentially unchanged during the study period (data not shown). Thus, we found no evidence in this investigation of hypophosphasia carriers that PS is a physiological substrate for TNSALP or PALP.

Our investigation of hypophosphatasia carriers indicates that PEA, PPi, and PLP are physiological substrates not only for TNSALP but for PALP as well. Possibly, all ALPs in nature can hydrolyze these phosphocompounds. Although in humans TNSALP acts critically in biomineralization, this promiscuous substrate specificity may reflect a role for ALPs both in the liberation of nonphosphate moieties as well as Pi. All ALP products of hydrolysis could then enter cells - a hypothesis that was proposed after study of yeast in the early part of this century (1).

\section{Acknowledgments}

We are grateful to the nursing and technical staff of the Metabolic Research Unit, Shriners Hospital for Crippled Children (St. Louis, MO) for making this study possible. Blood and urine specimens were kindly obtained by Beverly Otto, R. N. (West Palm Beach, FL), Dr. Paul Bennett (Carbondale, IL), and Dr. John Hucker (U. S. A. F. Hospital, Dover, DE). Frances C. Wilson provided expert secretarial help.

Supported, in part, by grants 15958 and 15963 from the Shriners Hospitals for Crippled Children (M. P. Whyte, K. N. Fedde); National Institute of Health grant AR-63956 (L. M. Ryan); and a Basil O'Connor Award from the March of Dimes (P. S. Henthorn). 


\section{References}

1. McComb, R. B., G. N. Bowers, and S. Posen. 1979. Alkaline Phosphatase. Plenum Publishing Corp., New York.

2. Whyte, M. P. 1989. Alkaline phosphatase: physiologic role explored in hypophosphatasia. In Bone and Mineral Research. Vol. 6. W. A. Peck, editor. Elsevier Science Publishers, B. V. (Biomedical Division), Amsterdam. 175-218.

3. Harris, H. 1990. The human alkaline phosphatases: what we know and what we don't know. Clin. Chim. Acta. 186:133-150.

4. Moss, D. W. 1992. Perspectives in alkaline phosphatase research. Clin. Chem. 38:2486-2492.

5. Henthorn, P. S., M. Raducha, Y. N. Edwards, M. J. Weiss, C. Slaughter, M. A. Lafferty, and H. Harris. 1987. Nucleotide and amino acid sequences of human intestinal alkaline phosphatase: close homology to placental alkaline phosphatase. Proc. Natl. Acad. Sci. USA. 84:1234-1238.

6. Millan, J. L., and T. Manes. 1988. Seminoma-derived Nagao isoenzyme is encoded by a germ-cell alkaline phosphatase gene. Proc. Natl. Acad. Sci. USA. 85:3024-3028.

7. Weiss, M. J., K. Ray, P. S. Henthorn, B. Lamb, T. Kadesch, and H. Harris 1988. Structure of the human liver/bone/kidney alkaline phosphatase gene. $J$. Biol. Chem. 263:12002-12010.

8. Whyte, M. P. 1994. Hypophosphatasia and the role of alkaline phosphatase in skeletal mineralization. Endocr. Rev. 15:439-461.

9. Whyte, M. P. 1995. Hypophosphatasia. In The Metabolic and Molecular Basis of Inherited Disease, Ed. 7. C. R. Scriver, A. L. Beaudet, W. S. Sly, and

D. Valle, editors. McGraw-Hill, New York. 4095-4111.

10. Caswell, A. M., M. P. Whyte, and R. G. Russell. 1991. Hypophosphatasia and the extracellular metabolism of inorganic pyrophosphate: clinical and laboratory aspects. Crit. Rev. Clin. Lab. Sci. 28:175-232.

11. Weiss, M. J., D. E. Cole, K. Ray, M. P. Whyte, M. A. Lafferty, R. A. Mulivor, and H. Harris. 1988. A missense mutation in the human liver/bone/ kidney alkaline phosphatase gene causing a lethal form of hypophosphatasia. Proc. Natl. Acad. Sci. USA. 85:7666-7669.

12. Henthorn, P. S., M. Raducha, K. N. Fedde, M. A. Lafferty, and M. P. Whyte. 1992. Different missense mutations at the tissue-nonspecific alkaline phosphatase gene locus in autosomal recessively inherited forms of mild and severe hypophosphatasia. Proc. Natl. Acad. Sci. USA. 89:9924-9928.

13. Greenberg, C. R., C. L. D. Taylor, J. C. Haworth, L. E. Seargeant, S. Phillips, B. Triggs-Raine, and B. N. Chodirker. 1993. A homoallelic Gly ${ }^{317} \rightarrow$ Asp mutation in ALPL causes the perinatal (lethal) form of hypophosphatasia in Canadian Mennonites. Genomics. 17:215-217.

14. Kim, E. E., and H. W. Wyckoff. 1991. Reaction mechanism of alkaline phosphatase based on crystal structures. Two metal ion catalysis. J. Mol. Biol. 218:449-464.

15. Hulett, F. M., E. E. Kim, C. Bookstein, N. V. Kapp, C. W. Edwards, and H. W. Wyckoff. 1991. Bacillus subtilis alkaline phosphatases II and IV: cloning, sequencing, and comparisons of deduced amino acid sequence with Escherichia coli alkaline phosphatase three-dimensional structure. J. Biol. Chem. 266:10771084.

16. Henthorn, P. S., and M. P. Whyte. 1992. Missense mutations of the tissue nonspecific alkaline phosphatase gene in hypophosphatasia. Clin. Chem. 38:2501 2505 .

17. Whyte, M. P., S. L. Teitelbaum, W. A. Murphy, M. Bergfeld, and L. V. Avioli. 1979. Adult hypophosphatasia: clinical, laboratory, and genetic investigation of a large kindred with review of the literature. Medicine (Baltimore). 58:329-347.

18. Whyte, M. P., W. A. Murphy, and M. D. Fallon. 1982. Adult hypophosphatasia with chondrocalcinosis and arthropathy: variable penetrance of hypophosphatasemia in a large Oklahoma kindred. Am. J. Med. 72:631-641.

19. Silverman, J. L. 1962. Apparent dominant inheritance of hypophosphatasia. Arch. Intern. Med. 110:191-198.

20. Eastman, J. R., and D. Bixler. 1983. Clinical, laboratory and genetic investigations of hypophosphatasia: support for autosomal dominant inheritance with homozygous lethality. J. Crainofacial Genet. Dev. Biol. 3:213-234.

21. Cheung, C. P., and R. J. Suhadolnik. 1977. Analysis of inorganic pyrophosphate at the picomole level. Anal. Biochem. 83:61-63.

22. Mahuren, J. D., and S. P. Coburn. 1991. B 6 Vitamers: cation exchange HPLC. J. Nutr. Biochem. 1:659-663.

23. Mulivor, R. A., D. Bocelli, and H. Harris. 1985. Quantitative analysis of alkaline phosphatases in serum and amniotic fluid: comparison of biochemical and immunologic assays. J. Lab. Clin. Med. 105:342-348.

24. Chodirker, B. N., S. P. Coburn, L. E. Seargeant, M. P. Whyte, and C. R. Greenberg. 1990. Increased plasma pyridoxal-5'-phosphate levels in carriers of infantile hypophosphatasia before and after pyridoxine loading. J. Inherited Metab. Dis. 13:891-896.
25. Whyte, M. P., J. D. Mahuren, K. N. Fedde, F. S. Cole, and S. P. Coburn. 1988. Perinatal hypophosphatasia: tissue levels of vitamin $B_{6}$ are unremarkable despite markedly increased circulating concentrations of pyridoxal 5 '-phosphate (evidence for an ectoenzyme role for tissue nonspecific alkaline phosphatase). $J$. Clin. Invest. 81:1234-1239.

26. Robison, R. 1923. The possible significance of hexosephosphoric esters in ossification. Biochem. J. 17:286-293.

27. Simko, V. 1991. Alkaline phosphatases in biology and medicine. Dig. Dis. 9:189-209.

28. Chines, A., S. Coburn, D. Mahuren, M. Landt, L. Ryan, and M. P. Whyte. 1994. Hypophosphatasia: diagnostic sensitivity of assay for alkaline phosphatase, phosphoethanolamine, inorganic pyrophosphate, and pyridoxal 5'-phosphate. $J$. Bone Miner. Res. 9:S-425 a. (Abstr.)

29. Fedde, K. N., C. C. Lane, and M. P. Whyte. 1990. Alkaline phosphatase (tissue nonspecific isoenzyme) is a phosphoethanolamine and pyridoxal 5 '-phosphate ectophosphatase: normal and hypophosphatasia fibroblast study. Am. J. Hum. Genet. 47:767-775.

30. Makiya, R., L. E. Thornell, and T. Stigbrand. 1992. Placental alkaline phosphatase, a GPI-anchored protein, is clustered in clathrin-coated vesicles. Biochem. Biophys. Res. Commun. 183:803-808.

31. Millan, J. L., M. P. Whyte, L. V. Avioli, and W. H. Fishman. 1980. Hypophosphatasia (adult form): quantitation of serum alkaline phosphatase isoenzyme activity in a large kindred. Clin. Chem. 26:840-845.

32. Maxwell, D. J., K. Blau, R. D. Johnson, and R. J. Lilford. 1985. Activities of alkaline phosphatase in first trimester chorion biopsy tissue. Prenatal Diagn. 5:283-286.

33. Harris, H. 1980. The Principles of Human Biochemical Genetics. Ed. 3. Elsevier/North Holland, Amsterdam.

34. Abu-Hasan, N. S., and R. G. Sutcliffe. 1985. Placental alkaline phosphatase integrates via its carboxy-terminus into the microvillous membrane: its allotypes differ in conformation. Placenta. 6:391-404.

35. Birkett, D. J., J. Dowe, F. C. Neale, and S. Posen. 1966. Serum alkaline phosphatase in pregnancy: an immunological study. Br. Med. J. 5497:1210-1212.

36. Sussman, H. H., M. Bowman, and J. L. Lewis, Jr. 1968. Placental alkaline phosphatase in maternal serum during normal and abnormal pregnancy. Nature (Lond.). 218:359-360.

37. Contractor, S. F., E. Holmes-Ievers, A. Morgan, M. Oakey, and N. A. Staines. 1985. A monoclonal antibody based solid-phase enzyme-binding assay to measure levels of placental alkaline phosphatase in serum of women during pregnancy. J. Immunol. Methods. 79:99-108.

38. Rodin, A., A. Duncan, H. W. P. Quartero, G. Pistofidis, G. Mashiter, K. Whitaker, D. Crook, J. C. Stevenson, M. G. Chapman, and I. Fogelman. 1989. Serum concentrations of alkaline phosphatase isoenzymes and osteocalcin in normal pregnancy. J. Clin. Endocrinol. \& Metab. 8:1123-1127.

39. Valenzuela, G. J., L. A. Munson, N. M. Tarbaux, and J. R. Farley. 1987. Time-dependent changes in bone, placental, intestinal, and hepatic alkaline phosphatase activities in serum during human pregnancy. Clin. Chem. 33:1801-1806.

40. Sato, N., Y. Takahashi, and S. Asano. 1994. Preferential usage of the bone-type leader sequence for the transcripts of liver/bone/kidney-type alkaline phosphatase gene in neutrophilic granulocytes. Blood. 83:1093-1101.

41. Schenker, S., R. F. Johnson, J. D. Mahuren, G. I. Henderson, and S. P. Coburn. 1992. Human placental vitamin $B_{6}$ (pyridoxal) transport: normal characteristics and effects of ethanol. Am. J. Physiol. 262:R966-R974.

42. Fraser, D. 1957. Hypophosphatasia. Am. J. Med. 22:730-736.

43. Diczfalusy, E. 1974. Endocrine functions of the human fetus and placenta. Am. J. Obstet. Gynecol. 199:419-429.

44. Babuna, C., and E. Yemen. 1966. Enzymatic determination of placental function. Am. J. Obstet. Gynecol. 95:925-934.

45. Clubb, J. S., F. C. Neale, and S. Posen. 1965. The behavior of infused placental alkaline phosphatase in human subjects. J. Lab. Clin. Med. 66:493496.

46. Anderson, B. B., H. O'Brien, G. E. Griffin, and D. L. Mollin. 1980 Hydrolysis of pyridoxal 5 '-phosphate in plasma in conditions with raised alkaline phosphatase. Gut. 21:192-194.

47. Whyte, M. P., D. Habib, S. P. Coburn, F. Tecklenburg, L. Ryan, K. N. Fedde, and R. A. Stinson. 1992. Failure of hyperphosphatasemia by intravenous infusion of purified placental alkaline phosphatase to correct severe hypophosphatasia: evidence against a role for circulating ALP in skeletal mineralization. $J$. Bone Miner. Res. 7(Suppl. 1):S155a. (Abstr.)

48. Whyte, M. P., R. Valdes, Jr., L. M. Ryan, and W. H. McAlister. 1982. Infantile hypophosphatasia: enzyme replacement therapy by intravenous infusion of alkaline phosphatase-rich plasma from patients with Paget bone disease. $J$. Pediatr. 101:379-386.

49. Jung, A.; R. G. G. Russell, S. Bisaz, D. B. Morgan, and H. Fleish. 1970. Fate of intravenously injected pyrophosphate- ${ }^{32} \mathrm{P}$ in dogs. Am. J. Physiol. 218:1757-1764. 